\title{
TUBERCULOSIS
}

\section{Outbreak of isoniazid resistant tuberculosis in north London}

\author{
M C Ruddy, A P Davies, M D Yates, S Yates, S Balasegaram, Y Drabu, B Patel, S Lozewicz, S Sen, \\ M Bahl, E James, M Lipman, G Duckworth, J M Watson, M Piper, F A Drobniewski, H Maguire
}

Thorax 2004;59:279-285. doi: 10.1136/thx.2003.010405

See end of article for authors' affiliations

.....................

Correspondence to: Dr M C Ruddy, HPA Mycobacterium Reference Unit, King's College Hospital (Dulwich), Guy's King's and St Thomas' Medical School, London SE22 8QF, UK:

m.c.p.r@doctors.org.uk

Received 15 May 2003 Accepted 23 January 2004
Background: A description is given of a major outbreak of isoniazid monoresistant tuberculosis (TB) chiefly in north London, including prisons. The earliest case was diagnosed in 1995 with most cases appearing after 1999.

Methods: Confirmation of a local cluster of cases was confirmed by restriction fragment length polymorphism (RFLP IS61 10) typing or "rapid epidemiological typing" (RAPET). Further cases were found by retrospective analysis of existing databases, prospective screening of new isolates, and targeted epidemiological case detection including questionnaire analysis.

Results: By the end of 2001, 70 confirmed cases in London had been linked with a further 13 clinical cases in contacts and nine epidemiologically linked cases outside London. The epidemic curve suggests that the peak of the outbreak has not yet been reached. Cases in the outbreak largely belong to a social group of young adults of mixed ethnic backgrounds including several individuals from professional/business backgrounds. Compared with other cases of TB reported to the enhanced surveillance scheme in London during 1999-2001, the cases are more likely to be of white $(26 / 70(37 \%)$ v 1308/7666 (17\%)) or black Caribbean ethnicity (17/70 (24\%) v 312/7666 (4\%)), born in the UK (41/70 (59\%) v 1335/7666 (17\%)), and male (52/70 (74\%) v 4195/7666 (55\%)). Drug misuse and/or prison detention are factors common to many cases.

Conclusions: The investigation of the outbreak revealed significant problems on an individual patient and population based level including difficulties with contact tracing, compliance, and the risk of developing multidrug resistance. This incident has demonstrated the value of molecular strain typing in investigating an extensive outbreak of TB. This is the first documented outbreak involving a UK prison.
$T$ he number of tuberculosis (TB) notifications in England and Wales steadily increased from 1987 to 1999, especially in London which has seen a doubling of cases. In 1999 there were a total of 6143 notifications of which 2493 (41\%) were from London (PHLS data from Notifications of Infectious Diseases database). Levels have risen mainly in inner London, but large outbreaks have not been observed. One of the biggest studies-the 1995-1997 pan-London study ${ }^{1}$ - detected few clusters, the largest of which featured 12 cases mainly from Somalia. Overall results suggested that less than $15 \%$ of London cases had recent acquisition of disease, the majority being due to importation or reactivation of disease.

In January 2000 medical microbiologists at a hospital in north London identified four cases of smear positive pulmonary TB in young men from the local community, all diagnosed within 1 week. Three of these isolates were subsequently shown to be isoniazid monoresistant Mycobacterium tuberculosis. This unusual occurrence suggested a local cluster, so the isolates were typed by the Public Health Laboratory Service (PHLS) Mycobacterium Reference Unit (MRU) using IS6110 restriction fragment length polymorphism (RFLP) analysis. ${ }^{2}$ This revealed an indistinguishable 15 band pattern fingerprint in the isolates from the three cases. RFLP is accepted as the international "gold standard" for assessment of genetic relatedness of strains of $M$ tuberculosis. After extraction of DNA and enzyme cutting, the presence of five or more bands of genetic material permits computerised matching with higher numbers of bands allowing increased confidence. $^{2}$

Isoniazid resistance in England and Wales had been steady over the previous years at approximately $6 \%(7.5-8 \%$ in London), but at this London hospital the proportion of isoniazid resistant strains among initial isolates of $M$ tuberculosis increased from $8.2 \%$ to $14.7 \%$ between 1998 and 1999 (PHLS Mycobnet data).

A local Incident Control Committee was established and initially looked at cases of isoniazid monoresistant $M$ tuberculosis in patients at the north London hospital over the previous 5 years (1995-2000). These isolates were RFLP typed at the PHLS MRU and a further 11 cases with the same pattern were detected, one dating back to 1995. Microbiologists from neighbouring hospitals were informed and performed similar exercises. In the light of information suggesting more cases outside the original district, a PHLS/ Communicable Disease Surveillance Centre (CDSC) multidistrict Incident Control Committee was formed in June 2000. It became apparent that many of the cases were linked socially or had in common drug misuse or a prison association. A separate subgroup of the main Incident Control Committee investigated the prison links. This paper provides a description of the investigation of the outbreak and the control measures implemented.

\section{METHODS \\ Epidemiology}

Case finding

Molecular fingerprinting was extended retrospectively to all isoniazid monoresistant $M$ tuberculosis isolates received from hospitals in London since 1 January 1999, and since June 2000 all isoniazid monoresistant isolates of $M$ tuberculosis received by the MRU from London hospitals have been typed by RFLP IS6110 typing and/or by "rapid epidemiological typing" (RAPET), a new rapid screening molecular typing method developed at the PHLS MRU. ${ }^{3}$ The small number of isolates referred elsewhere in London for confirmation of 


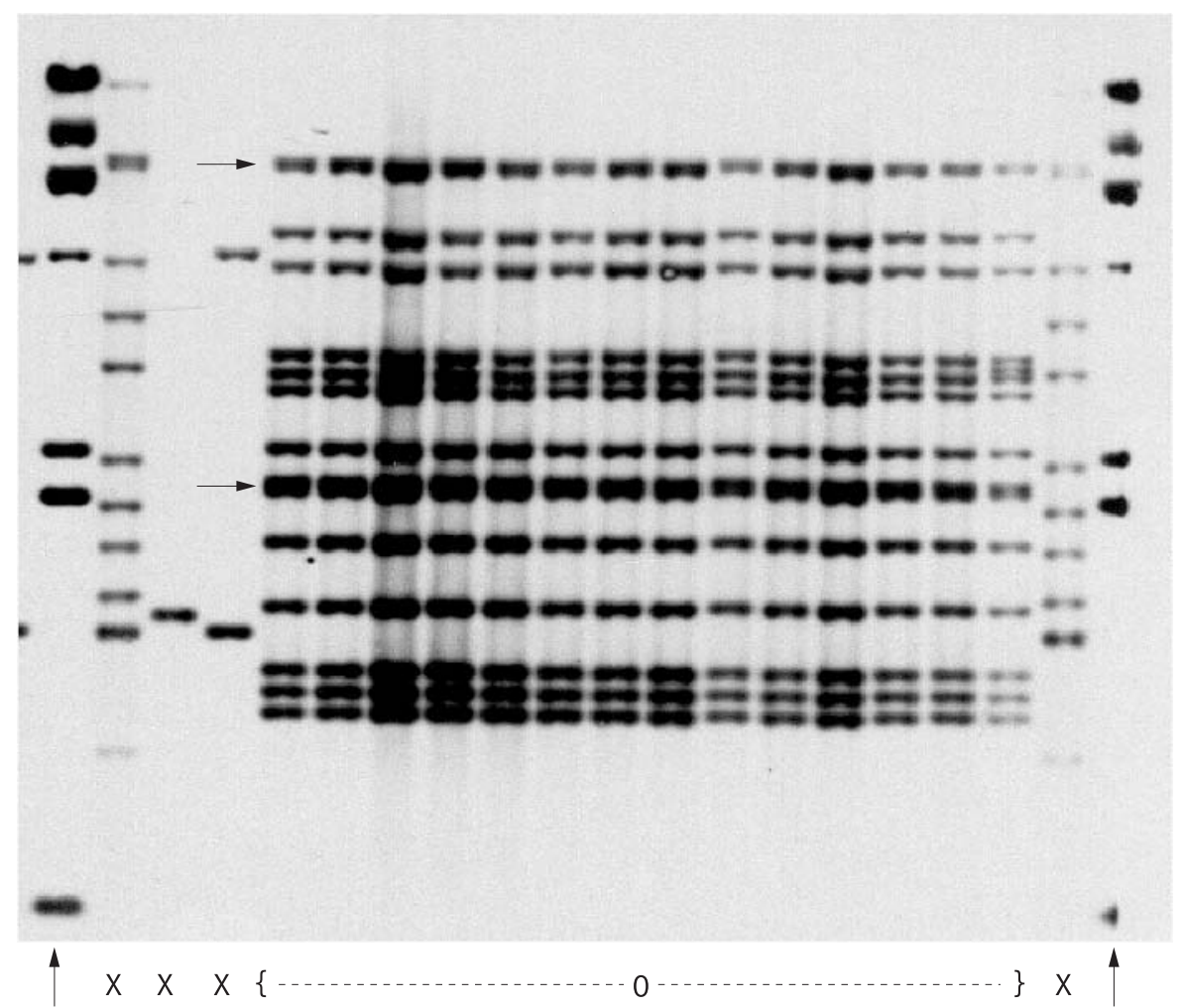

Figure 1 RFLP IS61 10 pattern of outbreak strain of M tuberculosis. Fourteen outbreak strain isolates (0) are shown with marker bands ( $\uparrow$ ) and four other non-outbreak isolates $(X)$ for comparison. The separation of the banding patterns into 15 distinct bands is seen against transmitted light, two bands merging on the photograph $(\rightarrow)$

identity and sensitivity testing as well as isolates from culture confirmed known contacts of cases outside London are also being subjected to RAPET and RFLP typing.

In addition, a review was carried out of databases of RFLP typed isolates from other studies including the 1995-1997 St George's/Royal Free/MRU/University of Surrey London-wide RFLP study ${ }^{1}$ which analysed all isolates from hospitals within the M25 motorway and the 1998 PHLS national study.

\section{Case definition}

For the purposes of the investigation the following case definitions were used:

- Confirmed: confirmed cases associated with the outbreak were defined as patients with isolates of $M$ tuberculosis resistant to isoniazid which on RFLP typing have the same 15 band pattern, diagnosed since January 1995, and who were resident in London at the time of the diagnosis.

- Probable: probable cases were defined as for confirmed cases, except that to date they have undergone RAPET analysis but await RFLP typing.

- Clinical: clinical cases associated with the outbreak were those with known epidemiological links to a confirmed or probable case, diagnosed since January 1995, and resident in London at the time of diagnosis. These cases were either culture negative, or culture positive with typing information still awaited.

- Other linked cases: these were known epidemiologically linked cases with culture confirmed isoniazid monoresistant TB of the same RAPET and RFLP type, diagnosed since January 1995 but not resident in London at the time of diagnosis.

Contacts of cases who were placed on chemoprophylaxis were not considered cases.

\section{Questionnaire}

A questionnaire was developed to enquire into sociodemographic and other factors, including contact with anyone with TB, travel abroad in the last 2 years, drug and alcohol abuse, imprisonment, and social venues frequented. Questionnaires were completed for all confirmed or probable cases by local clinic staff in face to face interviews with patients. Further detailed proformas have been developed to ask about contact details for each case and record the outcome of screening and treatment.

\section{Microbiology}

DNA typing was performed using two methods. Firstly, a more rapid PCR based screening method (RAPET) was performed which has proved to be reliable in detecting strains subsequently found to have the outbreak RFLP pattern. ${ }^{3}$ These were then confirmed by the gold standard IS6110 RFLP method ${ }^{2}$ which requires prior culture of organisms to enable sufficient DNA to be extracted. Gel compar software was used to compare the unique 15 band RFLP pattern obtained with existing databases in the UK. The RFLP fingerprints and RAPET profiles are shown in figs 1 and 2, respectively.

\section{Statistical analysis}

Using Epi-Info 6, $\chi^{2}$ tests were carried out to compare the proportions with certain demographic characteristics among outbreak cases with other London cases during 1999-2001.

\section{RESULTS}

During 1998-9 the number of initial isolates of $M$ tuberculosis from London hospitals overall rose by $11 \%$, while in hospitals located in the north central sector of London the rise in all isolates was $24 \%$ and at the index north London hospital there was a rise of $108 \%$. Approximately one fifth of the rise at this hospital was accounted for by isoniazid monoresistant 
A

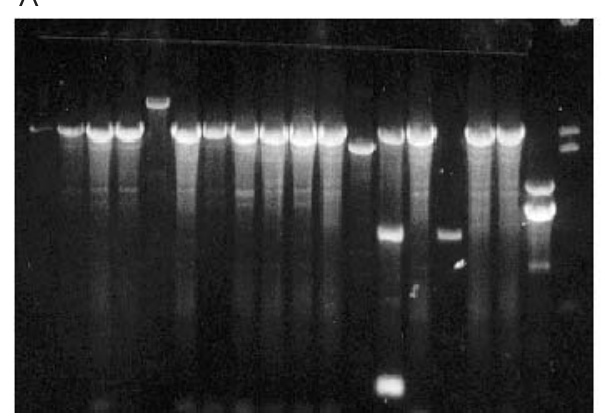

18 isolates

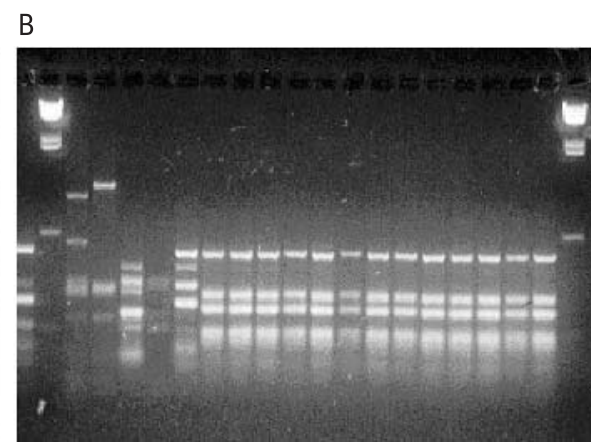

13 clustering

Figure 2 RAPET pattern of the outbreak strain of M tuberculosis (A) before and (B) after enzyme cutting with restriction enzyme Haell.

TB. Across London at the time of writing there were a total of 70 confirmed or probable cases of isoniazid monoresistant TB associated with this outbreak. Confirmed isolates $(n=55)$ had the same 15 band RFLP -IS6110 fingerprint (fig 1). Two of these cases (and a third linked case) were identified as a result of comparison with the previous existing RFLP databases of $M$ tuberculosis isolates in London.

All confirmed and probable cases exhibited the RAPET profiles shown in figs $2 \mathrm{~A}$ and $\mathrm{B}$. Figure $2 \mathrm{~B}$ shows the profile obtained after cutting PCR products with the enzyme HaeIII. A further 13 clinical cases, as well as nine linked cases not resident in London, have been diagnosed but are not discussed here.

One health district in north London comprising $6.8 \%$ of the London population accounts for 31 (44\%) of the confirmed or probable cases, with another focus at a north London prison in an adjacent health district. Most of the other cases lived elsewhere in north London with only six located in south London. The location of the cases, centred on the hospital of diagnosis, is shown in fig 3, superimposed on a map of the location of all cases of isoniazid resistant TB in London for comparison.

The earliest case was diagnosed in January 1995 in an individual born in Nigeria. The diagnosis dates of cases by quarter (fig 4) show the confirmed or probable cases arising from 1995 until December 2001 with no indication that the

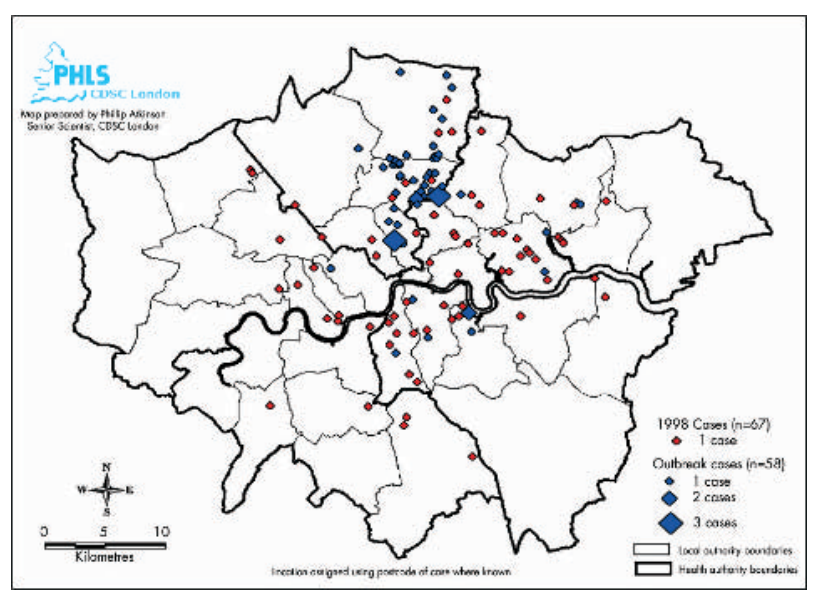

Figure 3 Map of London showing location of outbreak associated cases of isoniazid monoresistant tuberculosis where the full postcode is available superimposed on any isoniazid resistant cases in the PHLS/ DOH 1998 survey. rise has halted. A further 27 contacts (with other individuals under investigation) have been placed on chemoprophylaxis on the basis of positive skin testing and negative chest radiography.

\section{Age, sex and sociodemography}

Compared with other cases of TB in London reported to the national enhanced surveillance scheme during 1999-2002, patients were more likely to be male (52/70 (74\%) v 4195/ $7666(55 \%)$, odds ratio $(\mathrm{OR})=2.39,95 \%$ confidence interval (CI) 1.36 to 4.25$)$, white $(26 / 70$ (37\%) v 1308/7666 (17\%), OR $=2.8,95 \%$ CI 1.7 to 4.8$)$ or black Caribbean $(17 / 70(24 \%) v$ $312 / 7666(4 \%), \mathrm{OR}=7.56,95 \% \mathrm{CI} 4.2$ to 13.6$)$ in origin, and born in the UK $(41 / 70(59 \%) v 1335 / 7666(17 \%)$, OR $=6.7$, $95 \%$ CI 4.05 to 11.1 ). There were 18 females and 52 males among the confirmed or probable cases. The age range was 7-72 years with a median of approximately 28 years. However, the oldest case is likely to be the result of laboratory contamination in processing the original sample (see later).

\section{Country of birth and ethnic origin}

Forty one confirmed or probable cases were born in the UK, eight in Ireland, nine in Africa, one in Cyprus, one in Pakistan, one in Iraq, one in Jamaica, and eight unknown so far. The ethnic origin of the cases is summarised in table 1.

Occupations were varied and included schoolchildren $(n=2)$, factory workers, truck drivers $(n=2)$, hairdressers $(\mathrm{n}=2)$, a prison worker, a sex worker, a drug smuggler, and businessmen $(n=3)$. The HIV status of many of the cases was not assessed, their diagnosis having been made before the introduction of routine counselling for HIV testing in cases of TB.

\section{Site of infection}

Sixty two of the confirmed or probable cases (89\%) had pulmonary disease of which at least $28(40 \%)$ were sputum smear positive cases (the smear status of eight cases was unknown). This is a higher than expected proportion of pulmonary disease compared with all cases of TB generally in London (4168/7666 (54\%), OR $=14.25,95 \%$ CI 6.6 to 32.2 ) with a higher rate of sputum smear positivity (1318/4168 $(32 \%)$, OR $=1.44,95 \%$ CI 0.87 to 2.39$)$. Of the eight extrapulmonary cases of TB, four had tuberculosis lymphadenitis, two vertebral osteomyelitis, one soft tissue abscesses, and one meningitis.

\section{Epidemiological connections and risk factors}

Figure 5 shows the epidemiological connections established between the cases. Each case is assigned a number (referred 


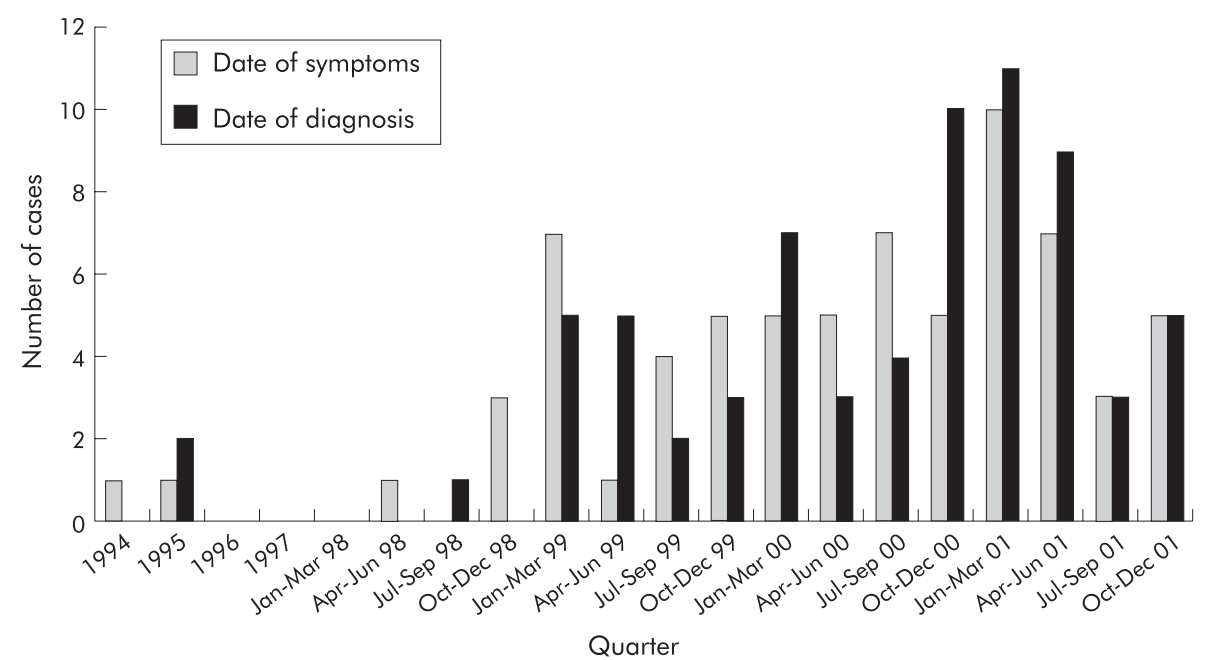

Figure 4 Isoniazid resistant tuberculosis outbreak: epidemic curve by quarter.

to below) and grouped by colour coding. Linked cases (those outside London) are also shown for completeness and are indicated by a boxed number. The linked cases are not considered in the discussion below.

Of the isolates typed retrospectively, the earliest found was from a case diagnosed in 1995 (case 14). This man had originally come from Nigeria as a student the previous year and he, together with 18 others grouped in red (cases 28,38 , $19,13,15,11,10,46,8,12,3,2,18,17,54,59,75$ and 1 ), were socially linked through a group of young adults in the north London area, a high proportion of whom $(8 / 19,42 \%)$ reported recreational drug use.

Another common feature of this group of 19 individuals was a prison contact. Four were or had been in custody in a

\begin{tabular}{|ll|}
\hline $\begin{array}{l}\text { Table } 1 \text { Epidemiology of } 70 \text { confirmed and } \\
\text { probable cases of isoniazid monoresistant } \\
\text { outbreak strain of M tuberculosis }\end{array}$ \\
\hline Sex & 52 M, 18 F \\
Age & Range 7-72 years (median 28) \\
Country of birth & 50 \\
Europe & 41 \\
UK & 8 \\
Ireland & 1 \\
Cyprus & 9 \\
Africa & 2 \\
Nigeria & 2 \\
Somalia & 2 \\
Kenya & 1 \\
Uganda & 1 \\
Angola & 1 \\
Mauritius & 3 \\
Elsewhere & 1 \\
Iraq & 1 \\
Jamaica & 1 \\
Pakistan & 8 \\
Unknown & \\
Ethnicity & 26 (including 7 Irish, 2 Turkish \\
White & Cypriot, 1 Greek Cypriot, 1 \\
& 17 \\
Black Caribbean & 15 \\
Black African & 2 (1 Indian, 1 Pakistani) \\
South Asian & 1 \\
Mixed race & 9 \\
Unknown & \\
\hline & \\
\hline & \\
\hline
\end{tabular}

London prison (cases 12, 8, 10, and 1). A further 10 of the 70 confirmed or probable cases had also been detained at the same prison grouped in blue (cases 48, 56, 36, 31, 47, 34, 39, $7,42,61$ ), in addition to one staff member at the prison (case 21). Three others who had not been in prison associated with this group in the community (cases $51,44,53$ ). One of these latter individuals (case 53) was admitted to the north London hospital at the centre of the outbreak and was probably infected through contact with one of the ex-prisoners, an infectious smear positive case who refused to stay in an isolation room on the same ward (case 39). The other two were the girlfriends of two of the prisoners. Another of the ex-prisoners (case 7) is a known injecting drug user and a further seven confirmed or probable cases outside the prison are believed to be linked to him via a "drug den" in north London grouped in yellow (cases 29, 33, 40, 52, 60, 79, 78). There are a further five confirmed/probable cases who are known drug users and may also be related to this group via this north London drug den, but this has not been proven (cases 66, 70, 71, 72, 73). The prison aspects are considered in more detail in a companion paper by Balasegaram et al (submitted for publication).

Two further cases grouped in purple (cases 63, 65) were members of staff in accident and emergency departments through which many of the smear positive patients had passed, raising the possibility of further unrecognised healthcare associated transmission.

Three other cases (cases 9, 6, 26) with no established links to any of the above social, prison, or drug using groups are known to be associated with one another; one of these spent time in prisons other than the institution above. A further two cases (cases 24,23) are contacts of one another through an association with the music industry (grouped in pink), but again with no established links to any of the above groups. Another two cases (cases 49, 25) are a brother and sister from south London, the sister having been a student in north London in 1998-9 and the brother having been a member of a music band but with no obvious contact with the above groups. There are also a husband and wife from South London, the husband born in Kenya (cases 77, 76).

Of the remaining confirmed or probable cases, five (cases $30,37,32,20,55$ ) are from Africa (one each from Nigeria, Uganda, and Kenya and two from Somalia) and a further case (case 64) was born in the UK of Nigerian parents. Two were alcohol abusers who lived in north London (cases 69, 


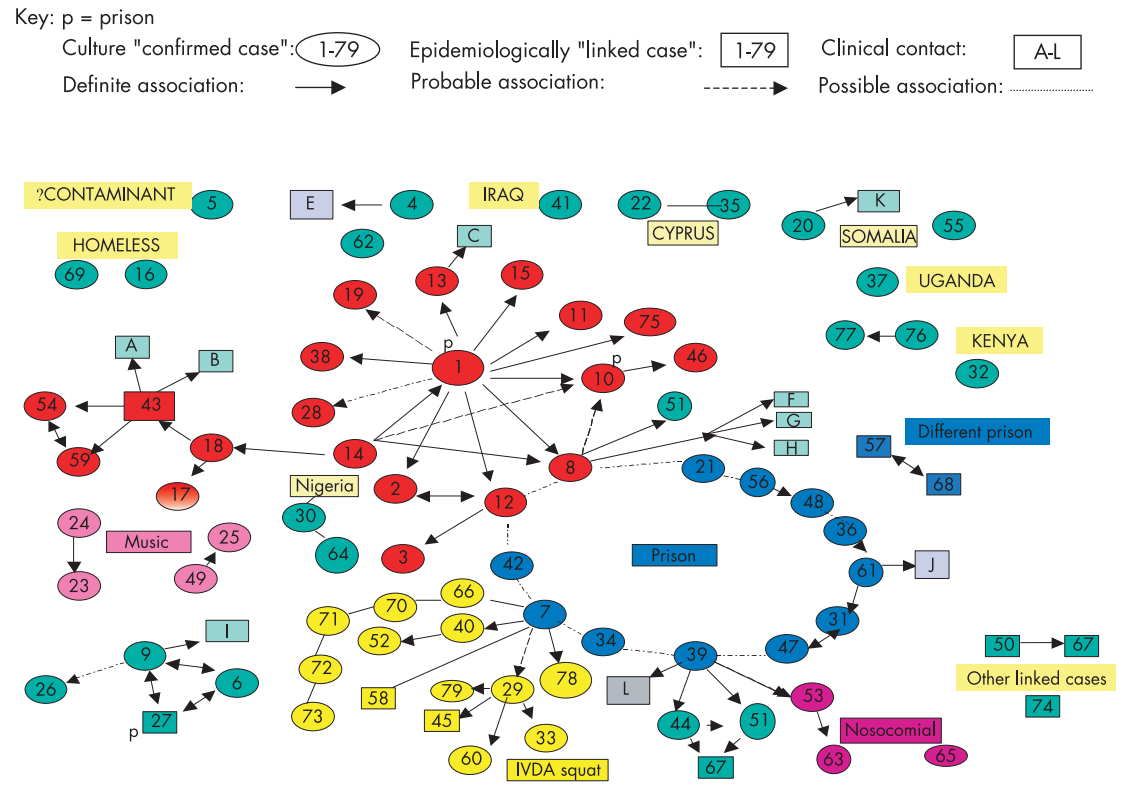

Figure 5 "Spider" chart of epidemiological associations.

16), one was a Turkish Cypriot student in north London (case 35), two (cases 22,41) were businessmen (an Iraqi and a Greek Cypriot), and two were black Caribbeans (cases 4, 62). Finally one case (case 5) is a retired lady with no obvious links to the other groups and it is strongly suspected that her isolate is a laboratory cross contamination from one of the other cases which was known to have been processed in the same laboratory on the same day as another isolate from a confirmed case. In the 1995-1997 pan-London study laboratory cross contamination was detected in $0.54 \%$ of microbiologically confirmed cases of $\mathrm{TB} .^{4}$

\section{Prison cases}

Sixteen of the 70 cases have been linked to a London prison and three others to prisons outside London. Investigations have revealed that transmission probably occurred in nine of these cases within the prison and possibly in one further case.

The first prison case with the outbreak strain was diagnosed with smear positive pulmonary disease in January 2000, shortly after release from the London prison. He had been symptomatic with a cough for the preceding 3 months while an inmate. A further six prisoners and one staff member who were in the prison during this symptomatic period were subsequently diagnosed with the outbreak strain. Two other prisoners who contracted the outbreak strain were in the prison during the presumed infectious periods of other cases and during readmissions of the first case. None of these had any prior history of TB or any contact with known cases of the outbreak strain outside the prison. The other cases with prison connections are believed to have contracted their disease outside the prison system through an established association with other known cases.

\section{Outcome of treatment}

To date, 30 of the 70 confirmed or probable cases are known to have completed treatment. Three have died; only one of the deaths was attributable to TB, one was a suicide, and the other was due to asthma. One child has been left with significant neurological deficits following tuberculosis meningitis. Of the remaining 37 cases receiving treatment, nine are very poorly compliant and four have been lost to follow up (one returned to Nigeria, the others are thought to be still living in London). One HIV positive case who has already had 16 months of treatment is hospitalised with cerebral tuberculomas and has developed concurrent resistance to rifampicin (multidrug resistant TB). Treatment compliance was a significant problem.

At least 17 of the cases (24\%) have demonstrated serious unreliability and poor adherence to the treatment regimen despite repeated follow up visits. In a further 10 cases (14\%) full compliance has been doubted by the TB nurses. Treatment compliance is therefore proving problematic in $38 \%$ of cases. This is compounded by the need for prolonged treatment, as recommended by the BTS for isoniazid resistant cases. $^{5}$

\section{Contact tracing}

There are at least 440 named close contacts of the confirmed or probable cases so far (an average of six per case but a small number of cases have a very large number of contacts-for example, one has declared 60).

Screening of a total of 269 close contacts so far completed has yielded 13 confirmed or probable cases, 13 clinical cases, and three linked cases. This represents a transmission rate of $11 \%$ among close contacts screened to date and suggests a high level of infection in close contacts. ${ }^{6}$ As mentioned above, one of the clinical cases occurred in a contact who was infected while an inpatient on a ward with one of the other confirmed cases. Furthermore, 27 infected contacts have been placed on chemoprophylaxis and 116 contacts are still in the process of being screened. Twenty one contacts have failed to attend any appointments, one is untraced, and 33 have been referred to health authorities outside London for screening.

In addition, extensive enhanced contact tracing is being performed in the prisons, schools, and workplaces. Total screening is expected to investigate over 1000 people.

\section{DISCUSSION}

The occurrence of 70 confirmed or probable cases, nine linked cases, and an additional 13 clinical cases with 27 further 
individuals receiving chemoprophylaxis suggests that there is a large outbreak of this strain in the London community, focused in north central London. The outbreak strain appears to have originated in Africa: the first diagnosed case in 1995 was from Nigeria and four other apparently unlinked cases are also African, including another Nigerian. The use of soft drugs was a feature common to many cases and some were involved with music, bands, or concerts. Other cases are relatively affluent professional and business people and would not normally be considered as being in a high risk group for TB-for example, a healthy 22 year old white student from an affluent social background who lived in north London in 1999 (case 25, fig 5) but had no known contact with any of the other cases.

\section{Virulence}

Some of the cases have become infected after apparently relatively brief contact with infectious cases-for instance, the member of staff working in the prison (case 21, fig 5) was estimated to have had no more than 20 minutes contact with any one prisoner in a week. This, together with the high proportion of infected contacts $(29 / 269(11 \%))$, seems to suggest that the strain may be "highly transmissible" according to BTS guidelines ${ }^{6}$ and raises the question of whether this strain of $M$ tuberculosis is a particularly infectious or pathogenic one. In 1998 an outbreak of 21 cases of TB (13 of them culture positive and confirmed by typing) was described in the USA where infection seemed to have occurred after very short periods of contact between cases. ${ }^{7}$ This strain of $M$ tuberculosis appeared to have a particularly high propensity for causing disease in mice compared with a reference strain. It has been reported previously that drug resistant strains of $M$ tuberculosis may be less infectious/ pathogenic than their drug susceptible counterparts ${ }^{8}$ (the mutations required to confer drug resistance possibly proving a "biological burden" on the organisms). This has particularly been described in the case of isoniazid resistance ${ }^{9}{ }^{10}$ as many of these strains have mutations in the $k a t G$ gene coding for catalase which is a virulence factor for $M$ tuberculosis. Targeted probing of the north London outbreak isolate failed to show mutations in the common kat $G$ sites. There was, however, a definite mutation in the inhA site which might not affect fitness in the same way.

\section{Handling an outbreak: managing patients and their contacts}

It is clearly important that the BTS guidelines ${ }^{56}$ are adhered to in the treatment of cases and that contacts should be followed up vigorously. Individual clinicians were contacted directly and advised of the recommendations of the Incident Control Committee, and information was also provided to chief executives of 35 hospital trusts and 16 health authorities across London. Full adherence with treatment is essential to minimise the risks of spreading disease in the community and of selecting multidrug resistance. At least one third of our cases had difficulty adhering to treatment and intensive input by staff, including a dedicated TB nurse employed specifically for the outbreak, was vital.

The case load associated with this incident placed greatly increased demands on nurses and chest physicians as well as on pharmacy, laboratory, and radiology personnel. The outbreak highlighted the need for urgent deployment of funds, greater flexibility, and cooperative local working. It has also demonstrated the lack of a clear and rapid system for the targeted allocation of additional resources from health funds to cope with local outbreaks. Without prompt directed funding it is often not possible to put in place control measures to "respond swiftly and consistently to outbreaks and epidemics" as advocated in recent Department of Health recommendations. ${ }^{11}$ Contact tracing in this outbreak was initially supported by research and development funding. NHS reforms regarding the implementation of the Chief Medical Officer's new strategy "Getting Ahead of the Curve" requires all the agencies involved to resolve the difficulties inherent within outbreaks of communicable disease. These include meeting resource needs, in particular the need for "surge capacity" to cope with such outbreaks.

\section{Adherence to treatment}

Responsible clinicians were asked to review the outcome of each case 1 year after starting treatment and also 1 year after completion of treatment to ensure relapse has not occurred. Problems persist in this cohort of difficult to treat patients.

A particular difficulty is that many hospitals are unable to prescribe antituberculous therapy to patients free of charge and some cannot provide patients with written prescriptions for the drugs which have to be obtained separately via the patient's GP. Providing drugs at the patient's clinic visit is likely to improve clinic attendance as well as treatment adherence. Other incentives which should be considered are the provision of food when patients attend for follow up and covering the cost of travel to the clinic. Although many hospitals will reimburse cost of travel afterwards to patients, TB nurses sometimes have to pay fares in advance for patients out of their own pockets. Asylum seekers are unable to collect reimbursement in some hospitals as proof that they are receiving state benefits is required. Provision of "Travelcards" might be helpful. This suggestion and the need for incentives/enablers to promote adherence was discussed in a report on TB control in London from the NHS Executive ${ }^{12}$ and a recent update. ${ }^{13}$ The use of such incentives has been shown to improve attendance in certain groups..$^{14} 15$

Attempts were made to use legislative powers to improve adherence, but this is an unwieldy and an impractical tool. In some patients where there were major concerns about adherence to treatment, they could not be detained under the relevant legislation (the Public Health (Control of Disease) Act 1984 sections 37 and 38) as it could not be proved that they were smear positive at the time. Even in cases known to be infectious at the time, the legislation was found to be too impractical to implement. A review of this legislation is urgently needed. Continued intermittent treatment could produce multidrug resistance which is a real risk for some patients and plans must be prepared for this eventuality. It may be that consideration should be given to the withdrawal of treatment from some of the most noncompliant patients.

\section{Prison issues}

It has been recognised in other settings that treatment difficulties arise in prison communities especially with isoniazid monoresistant TB as well as multidrug resistant TB. ${ }^{16} 17$ This community outbreak included the first documented case of transmission of TB in a UK prison and has highlighted several areas concerning the diagnosis and management of TB in the prison system which needed review. The findings and recommendations of the subcommittee for the prison component of the outbreak will be addressed in a separate publication. However, the main areas addressed are: consideration of standardised symptom assessment and possible radiological screening in high risk areas; provision of adequate isolation facilities with appropriate air extraction, particularly for suspected multidrug resistant and immunosuppressed cases; training in TB case detection and monitoring of treatment, including directly observed therapy which should be the standard practice in this population; enhanced tracking of TB patients through 
the prison system to allow constant awareness by medical staff of their condition and treatment; provision of adequate occupational health service screening to prison workers already accepted as being at increased exposure risk. ${ }^{6}$ Greater cooperation and liaison between the community communicable disease control teams and prison healthcare staff is needed to facilitate contact tracing and continuity of treatment of patients moving into and out of the prison system. ${ }^{18}$ A system whereby local TB nurses in-reach into the prison system from the community would be one way of achieving this.

At the London prison involved, the following improvements in TB control have already been instituted as a result of this outbreak: symptom screening of new prisoners, direct supervision of administration of all doses of treatment, active contact tracing of cases, designation and training of a lead TB nurse, and occupational health screening.

\section{Diagnostic methods and epidemiological typing}

Use of rapid molecular methods for diagnosing TB and rifampicin resistance could help to rationalise the use of prison facilities and minimise cross infection. ${ }^{18}$ More extensive use of typing methods on prison cases should be undertaken to elucidate the extent of transmission.

The vital role of DNA fingerprinting in mapping and unravelling the outbreak has been clearly shown. Many of the epidemiological links established would not have beenand, indeed, were not-discovered by routine contact tracing alone. It is possible that, if universal typing was available nationally, the links between all the out of London cases would have been discovered. The HPA currently offers an ondemand typing service for outbreak investigations and has adopted a platform of VNTR-MIRU supported by IS6110 and other methods. High throughput techniques do make prospective typing of all isolates feasible and should be considered. That TB transmission has occurred in UK prisons in the past seems beyond doubt. The availability of reliable typing methods to confirm such transmission could provide the impetus to institute improved control measures and the means to assess their effectiveness. The key initial identifying marker for this outbreak strain was isoniazid resistance. Had the strain been fully sensitive, it is likely that detection of the outbreak would have been significantly more delayed with the systems currently used. It is important to consider strategies for prompt detection of outbreaks of any strain of $M$ tuberculosis which could include the setting of "alarm thresholds" at local clinics dealing with TB for early awareness of higher than expected numbers of cases, together with consideration of more widespread typing.

\section{ACKNOWLEDGEMENTS}

The authors thank all the TB liaison nurses, chest physicians, microbiologists and CsCDC throughout London who assisted in the outbreak investigation, as well as the following individuals: Professor $\mathrm{J}$ Dale, University of Surrey, for assistance in comparing the RFLP pattern with the database from the 1995-7 London analysis; Dr Z Fang for assistance in comparing the RFLP pattern with the database from the PHLS 1998 National Study; Dr T Brown for microarray targeted probing for resistance genes; P Atkinson; for statistical analysis and provision of map; $\mathrm{J}$ Carless for providing data from the CDSC TB database; and J Herbert for providing data from Mycobnet.

\section{Authors' affiliations}

M C Ruddy, M D Yates, F A Drobniewski, HPA Mycobacterium

Reference Unit, King's College Hospital (Dulwich), Guy's King's and St Thomas' Medical School, London SE22 8QF, UK

A P Davies, Y Drabu, B Patel, North Middlesex University Hospital Trust, Medical Microbiology Department, London N18 1QX, UK

S Yates, S Lozewicz, North Middlesex University Hospital Trust, Respiratory Medicine, London N18 1QX, UK

S Sen, Enfield Primary Care Trust, Holbrook House, Barnet EN4 ODR, UK M Bahl, Islington Primary Care Trust, Insull Wing, London NW1 2U, UK E James, Barnet and Chase Farm Hospital NHS Trust, Medical Microbiology Department, Barnet Hospital, High Barnet EN5 3DY, UK M Lipman, Royal Free Hospital NHS Trust, Respiratory Medicine, London NW3 2QG, UK

S Balasegaram, G Duckworth, J M Watson, HPA Communicable Disease Surveillance Centre, London NW9 5EQ, UK

M Piper, Prison Health, Department of Health, London Wellington House Task Force, London SE1 8UG, UK

H Maguire, HPA Communicable Disease Surveillance Centre (London), London W2 3QR, and St George's Hospital Medical School, London SWI7 OQT, UK

\section{REFERENCES}

1 Maguire HM, Dale JW, McHugh TD, et al. Molecular epidemiology of tuberculosis in London 1995 to 1997 demonstrating low rate of active transmission. Thorax 2002;57:617-22.

2 van Embden JDA, Cave MD, Crawford JT, et al. Strain identification of Mycobacterium tuberculosis by DNA fingerprinting: recommendations for a standard methodology. J Clin Microbiol 1993:31:406-9.

3 Yates MD, Drobniewski FA, Wilson SM. Evaluation of a rapid PCR-based epidemiological typing method for routine studies of Mycobacterium tuberculosis. J Clin Microbiol 2002;40:712-4.

4 Ruddy M, McHugh TD, Dale JW, et al. Estimation of the rate of unrecognised cross contamination with Mycobacterium tuberculosis in London microbiology laboratories. J Clin Microbiol 2002;40:4100-4.

5 Joint Tuberculosis Committee of the British Thoracic Society. Chemotherapy and management of tuberculosis in the United Kingdom: recommendations 1998. Thorax 1998:53:536-48.

6 Joint Tuberculosis Committee of the British Thoracic Society. Control and prevention of tuberculosis in the United Kingdom: code of practice 2000. Thorax 2000;55:887-901.

7 Valway SE, Sanchez MPC, Shinnick TF, et al. An outbreak involving extensive transmission of a virulent strain of Mycobacterium tuberculosis. N Engl J Med 1998;338:633-9.

8 Davies AP, Billington OJ, Bannister BA, et al. Comparison of fitness of two isolates of 'Mycobacterium tuberculosis, one of which had developed multidrug resistance during the course of treatment. J Infect 2000;41:1-4.

9 Barnett M, Bushby SRM, Mitcheson DA. Tubercle bacilli resistant to isoniazid: virulence and response to treatment with isoniazid in guinea pigs and mice. Br J Exp Pathol 1953;34:568-81.

10 Middlebrook G, Cohn ML, Schaefer WB. Studies on isoniazid and tubercle bacilli. 111 . The isolation, drug-susceptibility and catalase-testing of tubercle bacilli from isoniazid-treated patients. Am Rev Tuberc 1954;70:852-72.

11 Department of Health. Getting ahead of the curve-a strategy for combating infectious diseases (including other aspects of health protection). London: Department of Health, 2002 (available at www.doh.gov.uk/cmo/ publications.htm).

12 Hayward A. Tuberculosis control in London-the need for change. A report for the Thames Regional Directors of Public Health. A discussion document. London: NHS Executive, December, 1998.

13 Iskander R, Wass T, Jones J, et al. TB control in London: next steps. London: NHS London Regional Office, March 2001.

14 Pilote L, Tulsky JP, Zolopa AR, et al. Tuberculosis prophylaxis in the homeless. A trial to improve adherence to referral. Arch Intern Med 1996;156:161-5.

15 Fujiwara PI, Larkin C, Frieden TR. Directly observed therapy in New York City. History, implementation, results and challenges. Clin Chest Med 1997; 18:135-48.

16 Coninx R, Mathieu C, Debacher M, et al. First-line tuberculosis therapy and drug-resistant Mycobacterium tuberculosis in prisons. Lancet 1999 20;353:969-73.

17 Kimerling ME. The Russian equation: an evolving paradigm in tuberculosis control. Int J Tuberc Lung Dis 2000;4(12 Suppl 2):5160-7.

18 Drobniewski $\mathbf{F}$. Tuberculosis in prisons - the forgotten plague. Lancet 1995;346:948-9.

19 Drobniewski FA. Diagnosing multi drug resistant TB in Britain. BMJ 1998;317:1263-4. 